\title{
Tumor Results Method
}

National Cancer Institute

\section{Source}

National Cancer Institute. Tumor Results Method. NCI Thesaurus. Code C117414.

The technique used to administer the tumor results assessment. 